\title{
Lymphocyte subsets, anthropometric measurements and body composition in European adolescents. The HELENA Study
}

\author{
F. Perez de Heredia ${ }^{1}$, S. Gómez-Martínez ${ }^{1}$, L. E. Díaz ${ }^{1}$, A. M. Veses ${ }^{1}$, O. Androutsos ${ }^{2}$, \\ J. Dallongeville ${ }^{3}$, S. De Henauw ${ }^{4}$, L. Censi ${ }^{5}$, L. A. Moreno ${ }^{6}$, G. Palacios ${ }^{7}$ and A. Marcos ${ }^{1}$, \\ on behalf of the Helena Study Group \\ ${ }^{1}$ Immunonutrition Research Group, Institute of Food Science, Technology and Nutrition (ICTAN) Spanish National \\ Research Council (CSIC), Madrid, Spain, ${ }^{2}$ Department of Nutrition and Dietetics, Harokopio University, Athens, Greece, \\ ${ }^{3}$ INSERM U557, INRA U1125, CNAM, University Paris 13, Centre de Recherche en Nutrition Humaine, 93017 Bobigny, \\ France, ${ }^{4}$ Ghent University, Ghent, Belgium, ${ }^{5}$ Istituto Nazionale di Ricerca per gli Alimenti e la Nutrizione (INRAN), Rome, \\ Italy, ${ }^{6}$ Escuela Universitaria de Ciencias de la Salud, Universidad de Zaragoza, Zaragoza, Spain and ${ }^{7}$ University
} Polytechnic of Madrid, Madrid, Spain

There is a close relationship between obesity and the immune system. Obese individuals have been shown to present higher immune cell counts, mainly of total leukocytes, but also of neutrophils, lymphocytes and monocytes ${ }^{(1)}$. The association between leukocyte count and obesity has been observed in children and adolescents as well ${ }^{(2,3)}$, and it could also occur with other cell types, e.g., $\mathrm{T}$ lymphocytes ${ }^{(4)}$.

We have recently found a significant relation between total leukocytes and body mass index (BMI) in a cohort of European adolescents participating in the HELENA-CSS (Healthy Lifestyle in Europe by Nutrition in Adolescence-Cross-Sectional Study) (unpublished data). For the present work we studied further the relationships between lymphocyte subset counts and body composition and anthropometric measurements in this adolescent cohort. Blood samples were collected after an overnight fast from 1,089 boys and girls (12.5-17.5 years). After applying exclusion criteria to rule out possible infections, data were analysed from 751 adolescents (47.5\% males). The sample was divided into three categories according to tertiles of standardized BMI values. Absolute and relative numbers of total $\mathrm{T}$ lymphocytes $\left(\mathrm{CD}^{+}\right)$, helper and cytotoxic $\mathrm{T}$ cells $\left(\mathrm{CD} 4^{+}\right.$and $\left.\mathrm{CD} 8^{+}\right)$, natural killer cells $\left(\mathrm{CD} 16^{+} 56^{+}\right)$, B lymphocytes $\left(\mathrm{CD} 19^{+}\right)$, and naïve and memory $\mathrm{T}\left(\mathrm{CD} 45 \mathrm{RA}^{+}\right.$and $\left.\mathrm{CD} 45 \mathrm{RO}^{+}\right)$cells were compared between groups by means of analysis of covariance (ANCOVA), adjusting for country, gender and age. Pearson correlations adjusted for country, gender, age and BMI were then calculated between lymphocyte subset counts and anthropometric measurements (height, weight, waist, waist-to-hip ratio, waist-to-height ratio), body fat mass and body fat percentage. Non-normally distributed variables were previously log-transformed.

Adolescents in the upper BMI tertile had higher numbers of total $(P<0.01)$, helper $(P<0.05)$ and cytotoxic $(P<0.01) \mathrm{T}$ lymphocytes, and natural killer cells $(P<0.01)$. They also presented higher counts of memory T cells $\left(\mathrm{CD} 3 \mathrm{RO}^{+}, \mathrm{CD} 4 \mathrm{RO}^{+}\right.$and $\mathrm{CD}^{+} \mathrm{RO}{ }^{+} ; P<0.01$ for all). In addition, waist-to-hip and waist-to-height ratios were positively correlated with circulating numbers of CD3 ${ }^{+}(P<0.001), \mathrm{CD} 4^{+}$, $\mathrm{CD}^{+}, \mathrm{CD}^{+}{ }^{+}(P<0.05), \mathrm{CD}^{2} \mathrm{RO}^{+}$and $\mathrm{CD} 4 \mathrm{RO}^{+}(P<0.001)$.

These results indicate that BMI and markers of central body fat distribution are positively associated with circulating values of several lymphocyte subsets in European adolescents. These relationships could contribute to the obesity-related low-grade inflammatory status.

1. Huang ZS, Chien KL, Yang CY, Tsai KS, Wang CH. Lipids (2001) 36(3), 237-245.

2. Hsieh CH, Pei D, Kuo SW, et al. Pediatr Int (2007) 49(6), 827-832.

3. Wu CZ, Hsiao FC, Lin JD, et al. Acta Diabetol (2010) 47, 65-71.

4. Zaldivar F, McMurray RG, Nemet D, et al. Int J Obes (Lond) (2006) 30(6), 906-911. 